\title{
Iterative methods for a linearly perturbed algebraic matrix Riccati equation arising in stochastic control ${ }^{\text {th }}$
}

\author{
Chun-Hua Guo
}

Department of Mathematics and Statistics, University of Regina, Regina, SK S4S 0A2, Canada

\begin{abstract}
We start with a discussion of coupled algebraic Riccati equations arising in the study of linear-quadratic optimal control problem for Markov jump linear systems. Under suitable assumptions, this system of equations has a unique positive semidefinite solution, which is the solution of practical interest. The coupled equations can be rewritten as a single linearly perturbed matrix Riccati equation with special structures. We study the linearly perturbed Riccati equation in a more general setting and obtain a class of iterative methods from different splittings of a positive operator involved in the Riccati equation. We prove some special properties of the sequences generated by these methods, and determine and compare the convergence rates of these methods. Our results are then applied to the coupled Riccati equations of jump linear systems. We obtain linear convergence of the Lyapunov iteration and the modified Lyapunov iteration, and confirm that the modified Lyapunov iteration indeed has faster convergence than the original Lyapunov iteration.
\end{abstract}

Keywords: Coupled algebraic Riccati equations; Linearly perturbed Riccati equation; Iterative methods; Convergence rate.

2000 MSC: 15A24; 65F30.

\section{Introduction}

In the study of linear-quadratic optimal control problem for Markov jump linear systems $[3,5,6,8]$ we need to solve the coupled algebraic Riccati equations

$$
A_{k}^{T} X_{k}+X_{k} A_{k}-X_{k} B_{k} R_{k}^{-1} B_{k}^{T} X_{k}+C_{k}^{T} C_{k}+\sum_{j=1}^{N} \lambda_{k j} X_{j}=0
$$

$k=1, \ldots, N$, where $A_{k} \in \mathbb{R}^{n \times n}, B_{k} \in \mathbb{R}^{n \times m}, C_{k} \in \mathbb{R}^{\ell \times n}$ and $R_{k}=R_{k}^{T} \in \mathbb{R}^{m \times m}$ is positive definite. The scalars $\lambda_{k j}$ are such that $\lambda_{k j} \geq 0, k \neq j$, and $\lambda_{k k}=-\sum_{j \neq k} \lambda_{k j}$. Actually, the matrix

$$
\Lambda=\left[\begin{array}{cccc}
\lambda_{11} & \lambda_{12} & \cdots & \lambda_{1 N} \\
\lambda_{21} & \lambda_{22} & \cdots & \lambda_{2 N} \\
\vdots & \vdots & \vdots & \vdots \\
\lambda_{N 1} & \lambda_{N 2} & \cdots & \lambda_{N N}
\end{array}\right]
$$

is the transition rate matrix associated with a Markov process.

For any $A \in \mathbb{C}^{n \times n}$, the transpose and the conjugate transpose of $A$ are denoted by $A^{T}$ and $A^{*}$, respectively. The spectrum of $A$ is denoted by $\sigma(A)$. We denote by $\mathbb{C}_{<}$(resp. $\mathbb{C}_{\leq}$) the set of complex numbers

\footnotetext{
This work was supported in part by a grant from the Natural Sciences and Engineering Research Council of Canada. Email address: chguo@math.uregina.ca (Chun-Hua Guo)
} 
with negative (resp. nonpositive) real parts. For any Hermitian matrices $X$ and $Y$, we write $X>Y$ (or $Y<X$ ) if $X-Y$ is positive definite and we write $X \geq Y$ (or $Y \leq X$ ) if $X-Y$ is positive semidefinite.

A solution $\left(X_{1}, \ldots, X_{N}\right)$ of the coupled equations (1) is said to be positive semidefinite if $X_{k} \geq 0$ for $k=1, \ldots, N$. For the existence of such solutions we need the concept of mean-square stability. Here we describe mean-square stability by one of its equivalent properties. Thus (see [3] for example) a matrix tuple $\mathcal{G}=\left(G_{1}, \ldots, G_{N}\right)$ is said to be mean-square stable if there exists $\mathcal{M}=\left(M_{1}, \ldots, M_{N}\right)$, with $M_{k}>0$ for $k=1, \ldots, N$, such that

$$
G_{k}^{T} M_{k}+M_{k} G_{k}+\sum_{j=1}^{N} \lambda_{k j} M_{j}<0, \quad k=1, \ldots, N .
$$

Let $\mathcal{A}=\left(A_{1}, \ldots, A_{N}\right), \mathcal{B}=\left(B_{1}, \ldots, B_{N}\right), \mathcal{C}=\left(C_{1}, \ldots, C_{N}\right)$. We say $(\mathcal{A}, \mathcal{B})$ is mean-square stabilizable if there is $\mathcal{K}=\left(K_{1}, \ldots, K_{N}\right)$ such that $\left(A_{1}-B_{1} K_{1}, \ldots, A_{N}-B_{N} K_{N}\right)$ is mean-square stable; $(\mathcal{C}, \mathcal{A})$ is meansquare detectable if there is $\mathcal{K}=\left(K_{1}, \ldots, K_{N}\right)$ such that $\left(A_{1}-K_{1} C_{1}, \ldots, A_{N}-K_{N} C_{N}\right)$ is mean-square stable. We now assume that $(\mathcal{A}, \mathcal{B})$ is mean-square stabilizable and $(\mathcal{C}, \mathcal{A})$ is mean-square detectable. By $[3$, Theorem 2.1], the coupled Riccati equations (1) has a unique positive semidefinite solution $\left(X_{1}, \ldots, X_{N}\right)$. Moreover, the solution is mean-square stabilizing in the sense that $\left(A_{1}-B_{1} R_{1}^{-1} B_{1}^{T} X_{1}, \ldots, A_{N}-B_{N} R_{N}^{-1} B_{N}^{T} X_{N}\right)$ is mean-square stable.

The equations (1) are often rewritten as

$$
\left(A_{k}+\frac{1}{2} \lambda_{k k} I\right)^{T} X_{k}+X_{k}\left(A_{k}+\frac{1}{2} \lambda_{k k} I\right)-X_{k} B_{k} R_{k}^{-1} B_{k}^{T} X_{k}+C_{k}^{T} C_{k}+\sum_{j=1, j \neq k}^{N} \lambda_{k j} X_{j}=0 .
$$

The benefit of doing this is that $\sum_{j=1, j \neq k}^{N} \lambda_{k j} X_{j} \geq 0$ whenever $X_{k} \geq 0$ for $k=1, \ldots, N$.

To simplify the notation, we let

$$
D_{k}=A_{k}+\frac{1}{2} \lambda_{k k} I, \quad S_{k}=B_{k} R_{k}^{-1} B_{k}^{T}, \quad Q_{k}=C_{k}^{T} C_{k} .
$$

So (3) becomes

$$
D_{k}^{T} X_{k}+X_{k} D_{k}-X_{k} S_{k} X_{k}+Q_{k}+\sum_{j=1, j \neq k}^{N} \lambda_{k j} X_{j}=0, \quad k=1, \ldots, N .
$$

Several iterative methods are available to compute the unique positive semidefinite solution of (5). Newton's method for (5) is

$$
\begin{aligned}
& \left(D_{k}-S_{k} X_{k}^{(i)}\right)^{T} X_{k}^{(i+1)}+X_{k}^{(i+1)}\left(D_{k}-S_{k} X_{k}^{(i)}\right)+\sum_{j=1, j \neq k}^{N} \lambda_{k j} X_{j}^{(i+1)}+X_{k}^{(i)} S_{k} X_{k}^{(i)}+Q_{k}=0, \\
& k=1, \ldots, N, i=0,1, \ldots
\end{aligned}
$$

The convergence of Newton's method is locally quadratic, but it may be time-consuming to compute the $n \times n$ matrices $X_{k}^{(i+1)}(k=1, \ldots, N)$ from (6) when $n$ is large.

The Lyapunov iteration for $(5)$

$$
\begin{aligned}
& \left(D_{k}-S_{k} X_{k}^{(i)}\right)^{T} X_{k}^{(i+1)}+X_{k}^{(i+1)}\left(D_{k}-S_{k} X_{k}^{(i)}\right)+\sum_{j=1, j \neq k}^{N} \lambda_{k j} X_{j}^{(i)}+X_{k}^{(i)} S_{k} X_{k}^{(i)}+Q_{k}=0, \\
& k=1, \ldots, N, i=0,1, \ldots
\end{aligned}
$$

has been studied in [5] and [6]. The matrices $X_{k}^{(i+1)}$ in (7) can be computed efficiently by the Bartels-Stewart algorithm [1]. 
The modified Lyapunov iteration for (5)

$$
\begin{aligned}
& \left(D_{k}-S_{k} X_{k}^{(i)}\right)^{T} X_{k}^{(i+1)}+X_{k}^{(i+1)}\left(D_{k}-S_{k} X_{k}^{(i)}\right)+\sum_{j=1}^{k-1} \lambda_{k j} X_{j}^{(i+1)} \\
& \quad+\sum_{j=k+1}^{N} \lambda_{k j} X_{j}^{(i)}+X_{k}^{(i)} S_{k} X_{k}^{(i)}+Q_{k}=0, \quad k=1, \ldots, N, i=0,1, \ldots,
\end{aligned}
$$

has been studied in [3] and [8], in an attempt to speed up the convergence of the Lyapunov iteration. Note that the matrices $X_{1}^{(i+1)}, X_{2}^{(i+1)}, \ldots, X_{N}^{(i+1)}$ in (8) can still be computed efficiently, in this order, by the Bartels-Stewart algorithm. Numerical experiments in [8] show that the modified Lyapunov iteration has faster convergence than the Lyapunov iteration. In this paper we will determine and compare the convergence rates of the iterations (7) and (8). We will be able to confirm that the modified Lyapunov iteration indeed has faster convergence than the Lyapunov iteration.

The next result about the modified Lyapunov iteration is a slight modification of [8, Theorem 2.1], which does not require the mean-square stabilizability of $(\mathcal{A}, \mathcal{B})$ and the mean-square detectability of $(\mathcal{C}, \mathcal{A})$.

Theorem 1. Let $\mathcal{R}_{k}\left(X_{1}, \ldots, X_{N}\right)=D_{k}^{T} X_{k}+X_{k} D_{k}-X_{k} S_{k} X_{k}+Q_{k}+\sum_{j=1, j \neq k}^{N} \lambda_{k j} X_{j}, k=1, \ldots, N$, where $D_{k}, S_{k}, Q_{k}$ are as in (4). Assume that there exist real symmetric matrices $\hat{X}_{k}, X_{k}^{(0)}, k=1, \ldots, N$, such that $\mathcal{R}_{k}\left(\hat{X}_{1}, \ldots, \hat{X}_{N}\right) \geq 0, X_{k}^{(0)} \geq \hat{X}_{k}, \mathcal{R}_{k}\left(X_{1}^{(0)}, \ldots, X_{N}^{(0)}\right) \leq 0$ and $\sigma\left(D_{k}-S_{k} X_{k}^{(0)}\right) \subset \mathbb{C}_{<}$for all $k=1, \ldots, N$. Then the sequence $\left\{\left(X_{1}^{(i)}, \ldots, X_{N}^{(i)}\right)\right\}$ defined by (8) has the following properties:

(i) $X_{k}^{(i)} \geq X_{k}^{(i+1)}, X_{k}^{(i)} \geq \hat{X}_{k}$ and $\mathcal{R}_{k}\left(X_{1}^{(i)}, \ldots, X_{N}^{(i)}\right) \leq 0, k=1, \ldots, N, i=0,1, \ldots$

(ii) $\sigma\left(D_{k}-S_{k} X_{k}^{(i)}\right) \subset \mathbb{C}_{<}, k=1, \ldots, N, i=0,1, \ldots$

(iii) The sequence $\left\{\left(X_{1}^{(i)}, \ldots, X_{N}^{(i)}\right)\right\}$ converges to a solution $\left(\tilde{X}_{1}, \ldots, \tilde{X}_{N}\right)$ of $(5)$, and $\tilde{X}_{k} \geq \hat{X}_{k}$ for $k=$ $1, \ldots, N$.

(iv) $\sigma\left(D_{k}-S_{k} \tilde{X}_{k}\right) \subset \mathbb{C}_{\leq}$for $k=1, \ldots, N$. If $\mathcal{R}_{k}\left(\hat{X}_{1}, \ldots, \hat{X}_{N}\right)>0$ for $k=1, \ldots, N$, then $\sigma\left(D_{k}-S_{k} \tilde{X}_{k}\right) \subset$ $\mathbb{C}_{<}$for $k=1, \ldots, \bar{N}$.

Remark 1. In Theorem 2.1 (i) of [8], the weaker result $\mathcal{R}_{k}\left(X_{1}^{(i)}, \ldots, X_{N}^{(i)}\right) \leq \sum_{j=1}^{k-1} \lambda_{k j}\left(X_{j}^{(i)}-X_{j}^{(i+1)}\right)$ was given. However, the stronger result that $\mathcal{R}_{k}\left(X_{1}^{(i)}, \ldots, X_{N}^{(i)}\right) \leq 0$ was essentially proved in [8]. In fact, by equations (18) and (19) of [8] we have

$$
\mathcal{R}_{k}\left(X_{1}^{(r)}, \ldots, X_{N}^{(r)}\right)=-\sum_{j=k+1}^{N} \lambda_{k j}\left(X_{j}^{(r-1)}-X_{j}^{(r)}\right)-\left(X_{k}^{(r-1)}-X_{k}^{(r)}\right) S_{k}\left(X_{k}^{(r-1)}-X_{k}^{(r)}\right) \leq 0
$$

for any $r \geq 1$.

For theoretical analysis of the coupled equations (5), it is more convinient to rewrite them into one equation. As in [3] and [8], we let

$$
\begin{aligned}
& D=\operatorname{diag}\left(D_{1}, \ldots, D_{N}\right), \quad S=\operatorname{diag}\left(S_{1}, \ldots, S_{N}\right), \quad Q=\operatorname{diag}\left(Q_{1}, \ldots, Q_{N}\right), \\
& X=\operatorname{diag}\left(X_{1}, \ldots, X_{N}\right), \quad \Pi(X)=\operatorname{diag}\left(\sum_{j \neq 1} \lambda_{1 j} X_{j}, \ldots, \sum_{j \neq N} \lambda_{N j} X_{j}\right) .
\end{aligned}
$$

So (5) becomes

$$
D^{T} X+X D-X S X+Q+\Pi(X)=0 .
$$

In the next section we will study (11) in a more general setting. Our results will cover iterations (6), (7), and (8) simultaneously. In particular, Theorem 1 above will be obtained as a special case of our general results. 


\section{Iterative solution of a linearly perturbed Riccati equation}

Let $\mathcal{H}$ be the linear space of all $p \times p$ Hermitian matrices over the field $\mathbb{R}$. We consider the equation

$$
\mathcal{R}(X)=D^{*} X+X D-X S X+Q+\Pi(X)=0,
$$

where $D, S, Q \in \mathbb{C}^{p \times p}, Q^{*}=Q, S^{*}=S, S \geq 0$, and $\Pi$ is a positive linear operator from $\mathcal{H}$ into itself, i.e., $\Pi(X) \geq 0$ whenever $X \geq 0$. The Riccati function $\mathcal{R}$ is thus a mapping from $\mathcal{H}$ into itself. Matrix Riccati equations of this type have been studied in $[2,4,7,12,13]$. A solution $X_{+}$of (12) is called maximal if $X_{+} \geq X$ for any solution $X$. The maximal solution is often the desired solution in applications.

The Fréchet derivative of $\mathcal{R}$ at a matrix $X \in \mathcal{H}$ is a linear operator $\mathcal{R}_{X}^{\prime}: \mathcal{H} \rightarrow \mathcal{H}$ given by

$$
\mathcal{R}_{X}^{\prime}(H)=(D-S X)^{*} H+H(D-S X)+\Pi(H) .
$$

Newton's method for the solution of (12) is

$$
X_{i+1}=X_{i}-\left(\mathcal{R}_{X_{i}}^{\prime}\right)^{-1} \mathcal{R}\left(X_{i}\right), \quad i=0,1, \ldots
$$

By (13), the iteration (14) is equivalent to

$$
\left(D-S X_{i}\right)^{*} X_{i+1}+X_{i+1}\left(D-S X_{i}\right)+\Pi\left(X_{i+1}\right)=-X_{i} S X_{i}-Q, \quad i=0,1, \ldots
$$

The spectrum of any linear operator $\mathcal{L}$ will be denoted by $\sigma(\mathcal{L})$. The following result, obtained in [2], shows that the maximal solution of (12) can be found by Newton's method under suitable conditions.

Theorem 2. Assume that there exist Hermitian matrices $\hat{X}$ and $X_{0}$ such that $\mathcal{R}(\hat{X}) \geq 0$ and $\sigma\left(\mathcal{R}_{X_{0}}^{\prime}\right) \subset \mathbb{C}_{<}$. Then the Newton sequence $\left\{X_{i}\right\}_{i=0}^{\infty}$ is well defined and the following are true:

(i) $X_{k} \geq X_{k+1}, \quad X_{k} \geq \hat{X}, \quad \mathcal{R}\left(X_{k}\right) \leq 0, \quad k \geq 1$.

(ii) $\sigma\left(\mathcal{R}_{X_{k}}^{\prime}\right) \subset \mathbb{C}_{<}, \quad k \geq 0$.

(iii) $\lim _{k \rightarrow \infty} X_{k}=X_{+}$is the maximal solution of (12).

(iv) $\sigma\left(\mathcal{R}_{X_{+}}^{\prime}\right) \subset \mathbb{C}_{\leq}$.

Note that the solution of the linear equation (15) is required in each step of the Newton iteration. The presence of the linear operator $\Pi$ on the left hand side will usually make solving this equation very expensive when $n$ is large. This observation has lead to the consideration of the iteration

$$
\left(D-S X_{i}\right)^{*} X_{i+1}+X_{i+1}\left(D-S X_{i}\right)=-\Pi\left(X_{i}\right)-X_{i} S X_{i}-Q, \quad i=0,1, \ldots,
$$

in [7].

The modified Lyapunov iteration (8) suggests that we decompose the positive operator $\Pi$ as $\Pi=\Phi+\Psi$, where $\Phi$ and $\Psi$ are also positive operators, and consider the iteration

$$
\left(D-S X_{i}\right)^{*} X_{i+1}+X_{i+1}\left(D-S X_{i}\right)+\Phi\left(X_{i+1}\right)=-\Psi\left(X_{i}\right)-X_{i} S X_{i}-Q, \quad i=0,1, \ldots
$$

If $\Phi=\Pi$ then we get the Newton iteration (15). If $\Phi=0$ then we get the iteration (16). However, other choices of $\Phi$ may produce more efficient iterations.

We note that iteration (17) can be rewritten as

$$
\left(D-S X_{i}\right)^{*}\left(X_{i+1}-X_{i}\right)+\left(X_{i+1}-X_{i}\right)\left(D-S X_{i}\right)+\Phi\left(X_{i+1}-X_{i}\right)=-\mathcal{R}\left(X_{i}\right), \quad i=0,1, \ldots
$$

To study the convergence behaviour of iteration (17), we need some results from [2].

We first note that $\mathcal{H}$ is a Hilbert space with the Frobenius inner product $\langle X, Y\rangle=\operatorname{trace}(X Y)$. For a linear operator $\mathcal{L}$ on $\mathcal{H}$, let $\rho(\mathcal{L})=\max \{|\lambda|: \lambda \in \sigma(\mathcal{L})\}$ denote the spectral radius, and $\beta(\mathcal{L})=\max \{\operatorname{Re}(\lambda)$ : $\lambda \in \sigma(\mathcal{L})\}$ the spectral abscissa. $\mathcal{L}$ is called stable if $\sigma(\mathcal{L}) \subset \mathbb{C}_{<}$, inverse positive if $\mathcal{L}^{-1}$ exists and is positive, resolvent positive if the operator $\alpha I-\mathcal{L}$ is inverse positive for all sufficiently large $\alpha>0$. 
Theorem 3. (see [2]) Let $\mathcal{L}: \mathcal{H} \rightarrow \mathcal{H}$ be resolvent positive and $\Pi: \mathcal{H} \rightarrow \mathcal{H}$ be positive. Then $\mathcal{L}+\Pi$ is also resolvent positive. Moreover, the following are equivalent.

(i) $\mathcal{L}+\Pi$ is stable.

(ii) $-(\mathcal{L}+\Pi)$ is inverse positive.

(iii) There exists $X>0$ such that $(\mathcal{L}+\Pi)(X)<0$.

(iv) $\mathcal{L}$ is stable and $\rho\left(\mathcal{L}^{-1} \Pi\right)<1$.

Theorem 4. (see [2]) If $\mathcal{L}: \mathcal{H} \rightarrow \mathcal{H}$ is resolvent positive, then $\beta(\mathcal{L}) \in \sigma(\mathcal{L})$ and there exists a nonzero matrix $V \geq 0$ such that $\mathcal{L}(V)=\beta(\mathcal{L}) V$.

As noted in [2], if $\mathcal{L}$ is resolvent positive, then the adjoint operator $\mathcal{L}^{*}$ is also resolvent positive and $\beta\left(\mathcal{L}^{*}\right)=\beta(\mathcal{L})$.

Lemma 5. (see [2]) For any $A \in \mathbb{C}^{p \times p}$, the linear operator $\mathcal{L}_{A}: \mathcal{H} \rightarrow \mathcal{H}$ defined by

$$
\mathcal{L}_{A}(H)=A^{*} H+H A
$$

is resolvent positive. The adjoint operator of $\mathcal{L}_{A}$ is given by $\left(\mathcal{L}_{A}\right)^{*}(H)=A H+H A^{*}$.

We are now ready to prove the following convergence result for iteration (17), which is an extension of Theorem 2.2 of [7].

Theorem 6. Assume that there exist Hermitian matrices $\hat{X}$ and $X_{0}$ such that $\mathcal{R}(\hat{X}) \geq 0, X_{0} \geq \hat{X}, \mathcal{R}\left(X_{0}\right) \leq$ 0 , and $\sigma\left(\mathcal{L}_{D-S X_{0}}+\Phi\right) \subset \mathbb{C}_{<}$. Then the iteration (17) defines a sequence $\left\{X_{k}\right\}$ such that

(i) $X_{k} \geq X_{k+1}, \quad X_{k} \geq \hat{X}, \quad \mathcal{R}\left(X_{k}\right) \leq 0, \quad k \geq 0$.

(ii) $\sigma\left(\mathcal{L}_{D-S X_{k}}+\Phi\right) \subset \mathbb{C}_{<}, \quad k \geq 0$.

(iii) $\lim _{k \rightarrow \infty} X_{k}=\tilde{X}$ is a solution of (12) and $\tilde{X} \geq \hat{X}$.

(iv) $\sigma\left(\mathcal{L}_{D-S \tilde{X}}+\Phi\right) \subset \mathbb{C}_{\leq}$. If $\mathcal{R}(\hat{X})>0$, then $\sigma\left(\mathcal{L}_{D-S \tilde{X}}+\Phi\right) \subset \mathbb{C}_{<}$.

Proof. We prove by induction that for each $i \geq 0, X_{i+1}$ is uniquely determined and

$$
X_{i} \geq X_{i+1}, \quad X_{i} \geq \hat{X}, \quad \mathcal{R}\left(X_{i}\right) \leq 0, \quad \sigma\left(\mathcal{L}_{D-S X_{i}}+\Phi\right) \subset \mathbb{C}_{<} .
$$

For $i=0$, we already have $X_{0} \geq \hat{X}, \mathcal{R}\left(X_{0}\right) \leq 0$, and $\sigma\left(\mathcal{L}_{D-S X_{0}}+\Phi\right) \subset \mathbb{C}_{<}$. By (18) with $i=0$, Lemma 5 and Theorem $3, X_{1}$ is uniquely determined and $X_{0} \geq X_{1}$. We now assume that $X_{k+1}$ is uniquely determined and (19) is true for $i=k(k \geq 0)$. By (17) with $i=k$, we have

$$
\begin{aligned}
& \left(D-S X_{k}\right)^{*}\left(X_{k+1}-\hat{X}\right)+\left(X_{k+1}-\hat{X}\right)\left(D-S X_{k}\right)+\Phi\left(X_{k+1}-\hat{X}\right) \\
= & -\Psi\left(X_{k}\right)-X_{k} S X_{k}-Q-D^{*} \hat{X}-\hat{X} D-\Phi(\hat{X})+X_{k} S \hat{X}+\hat{X} S X_{k} \\
= & -\Psi\left(X_{k}\right)-X_{k} S X_{k}-\mathcal{R}(\hat{X})+\Psi(\hat{X})-\hat{X} S \hat{X}+X_{k} S \hat{X}+\hat{X} S X_{k} \\
= & -\Psi\left(X_{k}-\hat{X}\right)-\left(X_{k}-\hat{X}\right) S\left(X_{k}-\hat{X}\right)-\mathcal{R}(\hat{X}) .
\end{aligned}
$$

So

$$
\left(D-S X_{k}\right)^{*}\left(X_{k+1}-\hat{X}\right)+\left(X_{k+1}-\hat{X}\right)\left(D-S X_{k}\right)+\Phi\left(X_{k+1}-\hat{X}\right) \leq-\mathcal{R}(\hat{X}) \leq 0 .
$$

Therefore, $X_{k+1} \geq \hat{X}$ by Theorem 3. To show that $\mathcal{L}_{D-S X_{k+1}}+\Phi$ is stable, we write $D-S X_{k+1}=$ $D-S X_{k}+S\left(X_{k}-X_{k+1}\right)$ and use (20) to get

$$
\begin{aligned}
& \left(D-S X_{k+1}\right)^{*}\left(X_{k+1}-\hat{X}\right)+\left(X_{k+1}-\hat{X}\right)\left(D-S X_{k+1}\right)+\Phi\left(X_{k+1}-\hat{X}\right) \\
\leq & -\Psi\left(X_{k}-\hat{X}\right)-\left(X_{k}-\hat{X}\right) S\left(X_{k}-\hat{X}\right)+\left(X_{k}-X_{k+1}\right) S\left(X_{k+1}-\hat{X}\right)+\left(X_{k+1}-\hat{X}\right) S\left(X_{k}-X_{k+1}\right) \\
= & -\Psi\left(X_{k}-\hat{X}\right)-\left(X_{k+1}-\hat{X}\right) S\left(X_{k+1}-\hat{X}\right)-\left(X_{k}-X_{k+1}\right) S\left(X_{k}-X_{k+1}\right) .
\end{aligned}
$$


So

$$
\left(\mathcal{L}_{D-S X_{k+1}}+\Phi\right)\left(X_{k+1}-\hat{X}\right) \leq-\left(X_{k}-X_{k+1}\right) S\left(X_{k}-X_{k+1}\right)
$$

We also have

$$
\left(\mathcal{L}_{D-S X_{k+1}}+\Phi\right)\left(X_{k+1}-\hat{X}\right) \leq-\Psi\left(X_{k}-\hat{X}\right)-\left(X_{k}-X_{k+1}\right) S\left(X_{k}-X_{k+1}\right),
$$

which will be needed later.

If $\mathcal{L}_{D-S X_{k+1}}+\Phi$ is not stable, we know from Theorem 4 that $\left(\mathcal{L}_{D-S X_{k+1}}+\Phi\right)^{*}(V)=\beta V$ for some nonzero $V \geq 0$ and some number $\beta \geq 0$. Therefore,

$$
\left\langle V,\left(\mathcal{L}_{D-S X_{k+1}}+\Phi\right)\left(X_{k+1}-\hat{X}\right)\right\rangle=\left\langle\beta V, X_{k+1}-\hat{X}\right\rangle \geq 0 .
$$

On the other hand, we have by (22) that

$$
\left\langle V,\left(\mathcal{L}_{D-S X_{k+1}}+\Phi\right)\left(X_{k+1}-\hat{X}\right)\right\rangle \leq-\left\langle V,\left(X_{k}-X_{k+1}\right) S\left(X_{k}-X_{k+1}\right)\right\rangle \leq 0 .
$$

Therefore,

$$
\left\langle V,\left(X_{k}-X_{k+1}\right) S\left(X_{k}-X_{k+1}\right)\right\rangle=0 .
$$

So, $\operatorname{trace}\left(V^{1 / 2}\left(X_{k}-X_{k+1}\right) S^{1 / 2} S^{1 / 2}\left(X_{k}-X_{k+1}\right) V^{1 / 2}\right)=0$. It follows that $S^{1 / 2}\left(X_{k}-X_{k+1}\right) V^{1 / 2}=0$ and thus $S\left(X_{k}-X_{k+1}\right) V=0$. Now, by Lemma 5 ,

$$
\begin{aligned}
\left(\mathcal{L}_{D-S X_{k}}+\Phi\right)^{*}(V) & =\left(D-S X_{k}\right) V+V\left(D-S X_{k}\right)^{*}+\Phi^{*}(V) \\
& =\left(\mathcal{L}_{D-S X_{k+1}}+\Phi\right)^{*}(V)+S\left(X_{k+1}-X_{k}\right) V+V\left(X_{k+1}-X_{k}\right) S \\
& =\left(\mathcal{L}_{D-S X_{k+1}}+\Phi\right)^{*}(V)=\beta V,
\end{aligned}
$$

which is contradictory to the stability of $\mathcal{L}_{D-S X_{k}}+\Phi$.

We have thus proved that $\mathcal{L}_{D-S X_{k+1}}+\Phi$ is stable. So, $X_{k+2}$ is uniquely determined and by (17) with $i=k+1$ and then with $i=k$ we get

$$
\begin{aligned}
& \left(D-S X_{k+1}\right)^{*}\left(X_{k+1}-X_{k+2}\right)+\left(X_{k+1}-X_{k+2}\right)\left(D-S X_{k+1}\right)+\Phi\left(X_{k+1}-X_{k+2}\right) \\
= & \left(D-S X_{k}+S\left(X_{k}-X_{k+1}\right)\right)^{*} X_{k+1}+X_{k+1}\left(D-S X_{k}+S\left(X_{k}-X_{k+1}\right)\right) \\
& +\Phi\left(X_{k+1}\right)+\Psi\left(X_{k+1}\right)+X_{k+1} S X_{k+1}+Q \\
= & -\Psi\left(X_{k}-X_{k+1}\right)-X_{k} S X_{k}+X_{k+1} S X_{k+1}+\left(X_{k}-X_{k+1}\right) S X_{k+1}+X_{k+1} S\left(X_{k}-X_{k+1}\right) \\
= & -\Psi\left(X_{k}-X_{k+1}\right)-\left(X_{k}-X_{k+1}\right) S\left(X_{k}-X_{k+1}\right) \leq 0 .
\end{aligned}
$$

Therefore, $X_{k+1} \geq X_{k+2}$. Since

$$
\left(D-S X_{k+1}\right)^{*}\left(X_{k+1}-X_{k+2}\right)+\left(X_{k+1}-X_{k+2}\right)\left(D-S X_{k+1}\right)+\Phi\left(X_{k+1}-X_{k+2}\right)=\mathcal{R}\left(X_{k+1}\right)
$$

by (18) with $i=k+1$, we have also obtained $\mathcal{R}\left(X_{k+1}\right) \leq 0$. The induction process is now complete. Thus, the sequence $\left\{X_{k}\right\}$ is well defined, monotonically decreasing, and bounded below by $\hat{X}$. Let $\lim _{k \rightarrow \infty} X_{k}=\tilde{X}$. We have $\tilde{X} \geq \hat{X}$. By taking limits in (17), we see that $\tilde{X}$ is a solution of (12). Since $\sigma\left(\mathcal{L}_{D-S X_{k}}+\Phi\right) \subset \mathbb{C}_{<}$ for each $k, \sigma\left(\mathcal{L}_{D-S \tilde{X}}+\Phi\right) \subset \mathbb{C}_{\leq}$. If $\mathcal{R}(\hat{X})>0$, then we have $\left(\mathcal{L}_{D-S \tilde{X}}+\Phi\right)(\tilde{X}-\hat{X})<0$ by letting $k \rightarrow \infty$ in $(21)$. If $\mathcal{L}_{D-S \tilde{X}}+\Phi$ is not stable, we have $\left(\mathcal{L}_{D-S \tilde{X}}+\Phi\right)^{*}(V)=\beta V$ for some nonzero $V \geq 0$ and some number $\beta \geq 0$. Therefore, $\left\langle V,\left(\mathcal{L}_{D-S \tilde{X}}+\Phi\right)(\tilde{X}-\hat{X})\right\rangle=\langle\beta V, \tilde{X}-\hat{X}\rangle \geq 0$. On the other hand, we have $\left\langle V,\left(\mathcal{L}_{D-S \tilde{X}}+\Phi\right)(\tilde{X}-\hat{X})\right\rangle<0$ since $\left(\mathcal{L}_{D-S \tilde{X}}+\Phi\right)(\tilde{X}-\hat{X})<0$. The contradiction shows that $\sigma\left(\mathcal{L}_{D-S \tilde{X}}+\Phi\right) \subset \mathbb{C}_{<}$

The next result is an extension of Theorem 2.8 of [7]. The assumption in Theorem 6 that $\sigma\left(\mathcal{L}_{D-S X_{0}}+\Phi\right) \subset$ $\mathbb{C}_{<}$is replaced by the stronger assumption that $\sigma\left(\mathcal{R}_{X_{0}}^{\prime}\right) \subset \mathbb{C}_{<}$. That $X_{0} \geq \hat{X}$ is no longer given as an assumption, but can be proved from other assumptions given. The conclusions (ii), (iii), (iv) in the next theorem are accordingly stronger than those in Theorem 6 . 
Theorem 7. Assume that there exist Hermitian matrices $\hat{X}$ and $X_{0}$ such that $\mathcal{R}(\hat{X}) \geq 0, \mathcal{R}\left(X_{0}\right) \leq 0$, and $\sigma\left(\mathcal{R}_{X_{0}}^{\prime}\right) \subset \mathbb{C}_{<}$. Then the iteration (17) defines a sequence $\left\{X_{k}\right\}$ such that

(i) $X_{k} \geq X_{k+1}, \quad X_{k} \geq \hat{X}, \quad \mathcal{R}\left(X_{k}\right) \leq 0, \quad k \geq 0$.

(ii) $\sigma\left(\mathcal{R}_{X_{k}}^{\prime}\right) \subset \mathbb{C}_{<}, \quad k \geq 0$.

(iii) $\lim _{k \rightarrow \infty} X_{k}=X_{+}$, the maximal solution of (12).

(iv) $\sigma\left(\mathcal{R}_{X_{+}}^{\prime}\right) \subset \mathbb{C}_{\leq}$.

Proof. By Theorem 2 on Newton's method, $X_{1}^{N}=X_{0}-\left(\mathcal{R}_{X_{0}}^{\prime}\right)^{-1} \mathcal{R}\left(X_{0}\right) \geq \hat{X}$. Since $\mathcal{R}\left(X_{0}\right) \leq 0$ and $-\mathcal{R}_{X_{0}}^{\prime}$ is inverse positive by Theorem 3 , we also have $X_{0} \geq X_{1}^{N}$. Thus $X_{0} \geq \hat{X}$. Since $\mathcal{R}_{X_{0}}^{\prime}$ is stable, we know from Theorem 3 that the operator $\mathcal{L}_{D-S X_{0}}+\Phi$ is also stable. Therefore, all conclusions of Theorem 6 are true. Since $\lim _{k \rightarrow \infty} X_{k}=\tilde{X} \geq \hat{X}$ and $\hat{X}$ can be any solution of (12), we have $\tilde{X}=X_{+}$. We have thus proved (i) and (iii) of the theorem. Since (iv) follows from (ii), we need only to prove (ii). Assuming that $\mathcal{R}_{X_{k}}^{\prime}$ is stable for some $k \geq 0$, we need to prove that $\mathcal{R}_{X_{k+1}}^{\prime}$ is also stable. If $\mathcal{R}_{X_{k+1}}^{\prime}$ is not stable, we know from Theorem 4 that $\left(\mathcal{R}_{X_{k+1}}^{\prime}\right)^{*}(V)=\beta V$ for some nonzero $V \geq 0$ and some number $\beta \geq 0$. Thus

$$
\left\langle V, \mathcal{R}_{X_{k+1}}^{\prime}\left(X_{k+1}-\hat{X}\right)\right\rangle=\left\langle\beta V, X_{k+1}-\hat{X}\right\rangle \geq 0 .
$$

On the other hand, we have by (23) that

$$
\begin{aligned}
\mathcal{R}_{X_{k+1}}^{\prime}\left(X_{k+1}-\hat{X}\right) & =\left(\mathcal{L}_{D-S X_{k+1}}+\Phi\right)\left(X_{k+1}-\hat{X}\right)+\Psi\left(X_{k+1}-\hat{X}\right) \\
& \leq-\Psi\left(X_{k}-X_{k+1}\right)-\left(X_{k}-X_{k+1}\right) S\left(X_{k}-X_{k+1}\right) \\
& \leq-\left(X_{k}-X_{k+1}\right) S\left(X_{k}-X_{k+1}\right),
\end{aligned}
$$

and then $\left\langle V, \mathcal{R}_{X_{k+1}}^{\prime}\left(X_{k+1}-\hat{X}\right)\right\rangle \leq-\left\langle V,\left(X_{k}-X_{k+1}\right) S\left(X_{k}-X_{k+1}\right)\right\rangle \leq 0$. Therefore,

$$
\left\langle V,\left(X_{k}-X_{k+1}\right) S\left(X_{k}-X_{k+1}\right)\right\rangle=0 .
$$

So $S\left(X_{k}-X_{k+1}\right) V=0$ as before. Now, by Lemma 5 ,

$$
\begin{aligned}
\left(\mathcal{R}_{X_{k}}^{\prime}\right)^{*}(V) & =\left(D-S X_{k}\right) V+V\left(D-S X_{k}\right)^{*}+\Pi^{*}(V) \\
& =\left(\mathcal{R}_{X_{k+1}}^{\prime}\right)^{*}(V)+S\left(X_{k+1}-X_{k}\right) V+V\left(X_{k+1}-X_{k}\right) S \\
& =\left(\mathcal{R}_{X_{k+1}}^{\prime}\right)^{*}(V)=\beta V,
\end{aligned}
$$

which is contradictory to the stability of $\mathcal{R}_{X_{k}}^{\prime}$.

Remark 2. We have the following comments on Theorem 7 .

(a) If it is difficult to choose an $X_{0}$ with $\mathcal{R}_{X_{0}}^{\prime}$ stable and $\mathcal{R}\left(X_{0}\right) \leq 0$, by Theorem 2 we may get such an $X_{0}$ by applying one Newton iteration on a Hermitian matrix $X_{-1}$ such that $\mathcal{R}_{X_{-1}}^{\prime}$ is stable.

(b) By [2, Theorem 7.2], the conclusion (iv) in Theorem 7 can be strengthened to $\sigma\left(\mathcal{R}_{X_{+}}^{\prime}\right) \subset \mathbb{C}_{<}$if there is a Hermitian matrix $\hat{X}$ such that $\mathcal{R}(\hat{X})>0$.

For iteration (17), linear convergence can be guaranteed if $\mathcal{R}_{X_{+}}^{\prime}$ is stable. This will be a consequence of the following general result.

Theorem 8. (see [9, p. 21]) Let $T$ be a (nonlinear) operator from a Banach space $E$ into itself and $x^{*} \in E$ be a solution of $x=T x$. If $T$ is Fréchet differentiable at $x^{*}$ with $\rho\left(T_{x^{*}}^{\prime}\right)<1$, then the iterates $x_{k+1}=T x_{k}(k=0,1, \ldots)$ converge to $x^{*}$, provided that $x_{0}$ is sufficiently close to $x^{*}$. Moreover, for any $\epsilon>0$,

$$
\left\|x_{k}-x^{*}\right\| \leq c\left(x_{0} ; \epsilon\right)\left(\rho\left(T_{x^{*}}^{\prime}\right)+\epsilon\right)^{k}
$$

where $\|\cdot\|$ is the norm in $E$ and $c\left(x_{0} ; \epsilon\right)$ is a constant independent of $k$. 
Theorem 9. Let the sequence $\left\{X_{k}\right\}$ be as in Theorem \%. If $\mathcal{R}_{X_{+}}^{\prime}$ is stable, then

$$
\limsup _{k \rightarrow \infty} \sqrt[k]{\left\|X_{k}-X_{+}\right\|} \leq \rho\left(-\left(\mathcal{L}_{D-S X_{+}}+\Phi\right)^{-1} \Psi\right)<1,
$$

where $\|\cdot\|$ is any matrix norm.

Proof. The iteration (17) can be written as $X_{i+1}=F\left(X_{i}\right)$ with

$$
F(X)=\left(\mathcal{L}_{D-S X}+\Phi\right)^{-1}(-\Psi(X)-X S X-Q) .
$$

Routine computations yield

$$
F\left(X_{+}+H\right)-F\left(X_{+}\right)=-\left(\mathcal{L}_{D-S X_{+}}+\Phi\right)^{-1} \Psi(H)+o(H),
$$

where $o(H)$ denotes some matrix $W(H)$ with $\lim _{\|H\| \rightarrow 0} \frac{\|W(H)\|}{\|H\|}=0$. Therefore, the Fréchet derivative of $F$ at the matrix $X_{+}$is $F_{X_{+}}^{\prime}=-\left(\mathcal{L}_{D-S X_{+}}+\Phi\right)^{-1} \Psi$. Since $\mathcal{R}_{X_{+}}^{\prime}$ is stable, we have $\rho\left(\left(\mathcal{L}_{D-S X_{+}}+\Phi\right)^{-1} \Psi\right)<1$ by Theorem 3. Therefore,

$$
\limsup _{k \rightarrow \infty} \sqrt[k]{\left\|X_{k}-X_{+}\right\|} \leq \rho\left(-\left(\mathcal{L}_{D-S X_{+}}+\Phi\right)^{-1} \Psi\right)<1
$$

by Theorems 7 and 8 .

While we have $\lim \sup _{k \rightarrow \infty} \sqrt[k]{\left\|X_{k}-X_{+}\right\|} \leq \rho\left(-\left(\mathcal{L}_{D-S X_{+}}+\Phi\right)^{-1} \Psi\right)$ in Theorem 9, equality typically holds in situations like this. It is a common practice that the spectral radius is used to judge the rate of convergence for a generic starting matrix $X_{0}$. We will now examine the effect of the decomposition of the operator $\Pi$ on the rate of convergence.

Theorem 10. Consider two decompositions of the operator $\Pi$ : $\Pi=\Phi_{1}+\Psi_{1}$ and $\Pi=\Phi_{2}+\Psi_{2}$, where $\Phi_{1}, \Psi_{1}, \Phi_{2}, \Psi_{2}$ are positive operators. If $\mathcal{R}_{X_{+}}^{\prime}$ is stable and $\Psi_{1} \leq \Psi_{2}$, then

$$
\rho\left(-\left(\mathcal{L}_{D-S X_{+}}+\Phi_{1}\right)^{-1} \Psi_{1}\right) \leq \rho\left(-\left(\mathcal{L}_{D-S X_{+}}+\Phi_{2}\right)^{-1} \Psi_{2}\right)
$$

Proof. Let $\Gamma=-\mathcal{L}_{D-S X_{+}}-\Pi$. Then $\Gamma$ is inverse positive by Theorem 3 and $\Gamma=\Omega_{1}-\Psi_{1}=\Omega_{2}-\Psi_{2}$, where $\Omega_{k}=-\left(\mathcal{L}_{D-S X_{+}}+\Phi_{k}\right)$ are inverse positive for $k=1,2$. We need to show $\rho\left(\Omega_{1}^{-1} \Psi_{1}\right) \leq \rho\left(\Omega_{2}^{-1} \Psi_{2}\right)$.

Note that for $k=1,2$,

$$
\Omega_{k}^{-1} \Psi_{k}=\left(\Gamma+\Psi_{k}\right)^{-1} \Psi_{k}=\left(I+\Gamma^{-1} \Psi_{k}\right)^{-1} \Gamma^{-1} \Psi_{k}
$$

So the eigenvalues $\lambda^{(k)}$ of $\Gamma^{-1} \Psi_{k}$ and the eigenvalues $\mu^{(k)}$ of $\Omega_{k}^{-1} \Psi_{k}$ are related by $\mu^{(k)}=\frac{\lambda^{(k)}}{1+\lambda^{(k)}}$. Since the function $f(x)=\frac{x}{1+x}$ is increasing for $x \geq 0$, the largest real eigenvalue of $\Omega_{k}^{-1} \Psi_{k}$ corresponds to the largest real eigenvalue of $\Gamma^{-1} \Psi_{k}$. By the Perron-Frobenius theory (see [10, Theorem 2.1], [11, Theorem 7] or [2, Theorem 3.5]), we know that $\rho\left(\Gamma^{-1} \Psi_{k}\right)$ is the largest real eigenvalue of $\Gamma^{-1} \Psi_{k}$ and $\rho\left(\Omega_{k}^{-1} \Psi_{k}\right)$ is the largest real eigenvalue of $\Omega_{k}^{-1} \Psi_{k}$. Therefore,

$$
\rho\left(\Omega_{k}^{-1} \Psi_{k}\right)=\frac{\rho\left(\Gamma^{-1} \Psi_{k}\right)}{1+\rho\left(\Gamma^{-1} \Psi_{k}\right)} .
$$

When $0 \leq \Psi_{1} \leq \Psi_{2}$, we have $0 \leq \Gamma^{-1} \Psi_{1} \leq \Gamma^{-1} \Psi_{2}$. Thus $\rho\left(\Gamma^{-1} \Psi_{1}\right) \leq \rho\left(\Gamma^{-1} \Psi_{2}\right)$ (see [10, Theorem 4.2] or $\left[2\right.$, Theorem 3.5]), and then $\rho\left(\Omega_{1}^{-1} \Psi_{1}\right) \leq \rho\left(\Omega_{2}^{-1} \Psi_{2}\right)$ by $(24)$. 


\section{Application to coupled Riccati equations for jump linear systems}

The coupled Riccati equations have been writtten in the form of (11), which is a special case of (12) since we can allow $X$ in (11) to be any $N n \times N n$ Hermitian matrix with $X_{1}, \ldots, X_{N}$ being its $n \times n$ diagonal blocks. The operator $\Pi$ in (11) is then a positive operator from $\mathcal{H}$ into itself, where $\mathcal{H}$ is the linear space of all $N n \times N n$ Hermitian matrices over the field $\mathbb{R}$. When $X$ has the special form $X=\operatorname{diag}\left(X_{1}, \ldots, X_{N}\right)$, we have $\mathcal{R}(X)=\operatorname{diag}\left(\mathcal{R}_{1}\left(X_{1}, \ldots, X_{N}\right), \ldots, \mathcal{R}_{N}\left(X_{1}, \ldots, X_{N}\right)\right)$. With the assumptions in Theorem 1 about iteration (8), we can let $\hat{X}=\operatorname{diag}\left(\hat{X}_{1}, \ldots, \hat{X}_{N}\right)$ and $X^{(0)}=\operatorname{diag}\left(X_{1}^{(0)}, \ldots, X_{N}^{(0)}\right)$, and verify all assumptions in Theorem 6 .

Indeed, for iteration (8) the operator $\Phi$ is defined by

$$
\Phi(X)=\operatorname{diag}\left(\sum_{j<1} \lambda_{1 j} X_{j}, \ldots, \sum_{j<N} \lambda_{N j} X_{j}\right),
$$

and for $X=\operatorname{diag}\left(X_{1}, \ldots, X_{N}\right)$ we can show that $\sigma\left(\mathcal{L}_{D-S X}+\Phi\right) \subset \mathbb{C}_{<}$if and only if $\sigma\left(D_{k}-S_{k} X_{k}\right) \subset \mathbb{C}_{<}$for $k=1, \ldots, N$. Suppose $\sigma\left(\mathcal{L}_{D-S X}+\Phi\right) \subset \mathbb{C}_{<}$. Then $\sigma\left(\mathcal{L}_{D-S X}\right) \subset \mathbb{C}_{<}$by Theorem 3 and so $\sigma(D-S X) \subset \mathbb{C}_{<}$. Thus $\sigma\left(D_{k}-S_{k} X_{k}\right) \subset \mathbb{C}_{<}$for $k=1, \ldots, N$. In the other direction, we suppose that $\sigma\left(D_{k}-S_{k} X_{k}\right) \subset \mathbb{C}_{<}$ for $k=1, \ldots, N$, and need to show that $\lambda=\beta\left(\mathcal{L}_{D-S X}+\Phi\right)<0$. By Theorem 4 there is a nonzero matrix $V \geq 0$ such that $\left(\mathcal{L}_{D-S X}+\Phi\right) V=\lambda V$. Let the diagonal blocks of $V$ be $V_{1}, \ldots, V_{N}$ and let $r$ be the smallest integer such that $V_{r} \neq 0$. Then $\mathcal{L}_{D_{r}-S_{r} X_{r}} V_{r}=\lambda V_{r}$. Thus $\lambda<0$ since $\sigma\left(D_{r}-S_{r} X_{r}\right) \subset \mathbb{C}_{<}$.

Now we have all the conclusions in Theorem 6 . It is readily seen that each matrix $X^{(k)}$ has the form $X^{(k)}=\operatorname{diag}\left(X_{1}^{(k)}, \ldots, X_{N}^{(k)}\right)$. All conclusions in Theorem 1 follow immediately.

We also note that all conclusions in [8, Corollary 2.2] about iteration (7), where $\Phi=0$, follow from Theorem 6 directly.

We now assume that $(\mathcal{A}, \mathcal{B})$ is mean-square stabilizable and $(\mathcal{C}, \mathcal{A})$ is mean-square detectable, as in the first part of Section 1. So the coupled Riccati equations (1) has a unique positive semidefinite solution $\left(\tilde{X}_{1}, \ldots, \tilde{X}_{N}\right)$ and $\left(A_{1}-S_{1} \tilde{X}_{1}, \ldots, A_{N}-S_{N} \tilde{X}_{N}\right)$ is mean-square stable. Thus, there exists $\mathcal{M}=$ $\left(M_{1}, \ldots, M_{N}\right)$, with $M_{k}>0$ for $k=1, \ldots, N$, such that

$$
\left(A_{k}-S_{k} \tilde{X}_{k}\right)^{T} M_{k}+M_{k}\left(A_{k}-S_{k} \tilde{X}_{k}\right)+\sum_{j=1}^{N} \lambda_{k j} M_{j}=\left(D_{k}-S_{k} \tilde{X}_{k}\right)^{T} M_{k}+M_{k}\left(D_{k}-S_{k} \tilde{X}_{k}\right)+\sum_{j=1, j \neq k}^{N} \lambda_{k j} M_{j}<0
$$

for $k=1, \ldots, N$. Let $\tilde{X}=\operatorname{diag}\left(\tilde{X}_{1}, \ldots, \tilde{X}_{N}\right)$ and $M=\operatorname{diag}\left(M_{1}, \ldots, M_{N}\right)>0$. We then have $\left(\mathcal{L}_{D-S \tilde{X}}+\right.$ $\Pi)(M)<0$. By Theorem 3 we have $\sigma\left(\mathcal{R}_{\tilde{X}}^{\prime}\right)=\sigma\left(\mathcal{L}_{D-S \tilde{X}}+\Pi\right) \subset \mathbb{C}_{<}$.

To apply Theorem 7 , we can take $\hat{X}=0$ and any $X^{(0)}=\operatorname{diag}\left(X_{1}^{(0)}, \ldots, X_{N}^{(0)}\right) \geq 0$ with $\mathcal{R}\left(X_{0}\right) \leq 0$ and $\sigma\left(\mathcal{R}_{X_{0}}^{\prime}\right) \subset \mathbb{C}_{<}$. We conclude that $X^{(k)}=\operatorname{diag}\left(X_{1}^{(k)}, \ldots, X_{N}^{(k)}\right)$ converges to $X_{+}=\operatorname{diag}\left(\left(X_{+}\right)_{1}, \ldots,\left(X_{+}\right)_{N}\right) \geq$ 0 . So $\left(\left(X_{+}\right)_{1}, \ldots,\left(X_{+}\right)_{N}\right)=\left(\tilde{X}_{1}, \ldots, \tilde{X}_{N}\right)$, the unique positive semidefinite solution of (1). If iterations $(7)$ and (8) are used, then the convergence of either iteration is linear by Theorem 9 , and the convergence of (8) is faster by Theorem 10 .

We can also apply Theorem 6 for iterations (7) and (8), with $\hat{X}=0$ and any $X^{(0)}=\operatorname{diag}\left(X_{1}^{(0)}, \ldots, X_{N}^{(0)}\right) \geq$ 0 such that $\mathcal{R}\left(X_{0}\right) \leq 0$ and $\sigma\left(D_{k}-S_{k} X_{k}^{(0)}\right) \subset \mathbb{C}_{<}$for $k=1, \ldots, N$. In this case we have $\sigma\left(\mathcal{R}_{X_{k}}^{\prime}\right) \subset \mathbb{C}_{<}$for some $k \geq 0$ and we still have the above conclusions about iterations (7) and (8).

When the matrix $\Lambda$ in (2) more resembles an upper triangular matrix, we may use, instead of iteration (8), the following modified Lyapunov iteration

$$
\begin{aligned}
& \left(D_{k}-S_{k} X_{k}^{(i)}\right)^{T} X_{k}^{(i+1)}+X_{k}^{(i+1)}\left(D_{k}-S_{k} X_{k}^{(i)}\right)+\sum_{j=k+1}^{N} \lambda_{k j} X_{j}^{(i+1)} \\
& \quad+\sum_{j=1}^{k-1} \lambda_{k j} X_{j}^{(i)}+X_{k}^{(i)} S_{k} X_{k}^{(i)}+Q_{k}=0, \quad k=N, N-1, \ldots, 1, i=0,1, \ldots
\end{aligned}
$$


The results in Section 2 (Theorems $6,7,9,10$ ) can be applied to iteration (25) directly. In particular, the convergence of iteration (25) is also faster than that of iteration (7).

\section{References}

[1] R.H. Bartels and G.W. Stewart (1972). Solution of the matrix equation $A X+X B=C$. Comm. ACM 15: 820-826.

[2] T. Damm and D. Hinrichsen (2001). Newton's method for a rational matrix equation occurring in stochastic control. Linear Algebra Appl. 332-334: 81-109.

[3] J.B.R. do Val, J.C. Geromel, and O.L.V. Costa (1999). Solutions for the linear-quadratic control problem of Markov jump linear systems. J. Optim. Theory Appl. 103: 283-311.

[4] M.D. Fragoso, O.L.V. Costa, and C.E. de Souza (1998). A new approach to linearly perturbed Riccati equations arising in stochastic control. Appl. Math. Optim. 37: 99-126.

[5] Z. Gajic and I. Borno (1995). Lyapunov iterations for optimal control of jump linear systems at steady state. IEEE Trans. Automat. Control 40: 1971-1975.

[6] Z. Gajic and R. Losana (2000). Monotonicity of algebraic Lyapunov iterations for optimal control of jump parameter linear systems. Systems Control Lett. 41: 175-181.

[7] C.-H. Guo (2001). Iterative solution of a matrix Riccati equation arising in stochastic control. Oper. Theory Adv. Appl. 130: 209-221.

[8] I.G. Ivanov (2008). On some iterations for optimal control of jump linear equations. Nonlinear Analysis 69: $4012-4024$.

[9] M.A. Krasnoselskii, G.M. Vainikko, P.P. Zabreiko, Ya.B. Rutitskii, and V.Ya. Stetsenko (1972) Approximate Solution of Operator Equations, Wolters-Noordhoff Publishing, Groningen.

[10] I. Marek (1970). Frobenius theory of positive operators: Comparison theorems and applications. SIAM J. Appl. Math. 19: $607-628$.

[11] W.C. Rheinboldt and J.S. Vandergraft (1973). A simple approach to the Perron-Frobenius theory for positive operators on general partially-ordered finite-dimensional linear spaces. Math. Comp. 27: 139-145.

[12] E.I. Verriest and P. Florchinger (1995). Stability of stochastic systems with uncertain time delays. Systems Control Lett. 24: $41-47$.

[13] W.M. Wonham (1968). On a matrix Riccati equation of stochastic control. SIAM J. Control 6: 681-697. 